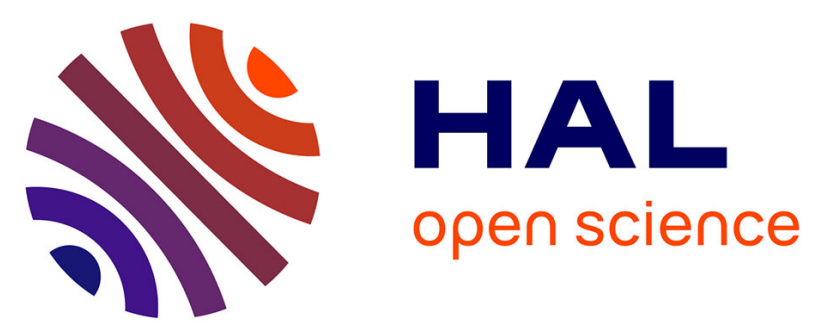

\title{
Combining Analytical and Simulation Approaches for Estimating End-to-End Delay in Multi-hop Wireless Networks
}

Francois Despaux, Ye-Qiong Song, Abdelkader Lahmadi

\section{- To cite this version:}

Francois Despaux, Ye-Qiong Song, Abdelkader Lahmadi. Combining Analytical and Simulation Approaches for Estimating End-to-End Delay in Multi-hop Wireless Networks. IEEE workshop WiSARN, in conjunction with IEEE 8th International Conference on Distributed Computing in Sensor Systems (DCOSS2012), IEE, May 2012, Hangzhou, China. pp.317-322. hal-00745243

HAL Id: hal-00745243

https://hal.inria.fr/hal-00745243

Submitted on 25 Oct 2012

HAL is a multi-disciplinary open access archive for the deposit and dissemination of scientific research documents, whether they are published or not. The documents may come from teaching and research institutions in France or abroad, or from public or private research centers.
L'archive ouverte pluridisciplinaire HAL, est destinée au dépôt et à la diffusion de documents scientifiques de niveau recherche, publiés ou non, émanant des établissements d'enseignement et de recherche français ou étrangers, des laboratoires publics ou privés. 


\section{Combining Analytical and Simulation Approaches for Estimating End-to-End Delay in Multi-hop Wireless Networks}

\author{
Francois Despaux \\ LORIA - University of Lorraine \\ France \\ francois.despauxeloria.fr
}

\author{
Ye-Qiong Song \\ LORIA - University of Lorraine \\ France \\ songeloria.fr
}

\author{
Abdelkader Lahmadi \\ LORIA - University of Lorraine \\ France \\ abdelkader. lahmadicloria.fr
}

\begin{abstract}
In this work, we present an empirical support of an analytical approach which employs a frequency domain analysis for estimating end-to-end delay in multi-hop networks. The proposed analytical results of the end-to-end delay distribution are validated through simulation and compared with queueing based analysis by defining two concrete scenarios. Our results demonstrate that an analytical prediction schema is insufficient to provide an adequate estimation of the end-to-end delay distribution function, but it requires to be combined with a simulation method for detailed links and nodes latencies distribution.
\end{abstract}

Index Terms-communication Reliability, end-to-end delay, latency, estimation, queueing delay.

\section{INTRODUCTION}

Multi-hop communication networks are widely deployed and their characteristics and design depend on the environment where they are placed as well as the tasks that their applications have to carry out. For many applications the fundamental requirement is a reliable end-to-end (e2e) communication. These applications usually rely on multi-hop networks where the e2e delay should taken into account in order to efficiently meet the application performance. Typically an application may require that the e2e delay should not exceed a given threshold with a certain probability, requiring thus a probabilistic QoS guarantee. Analyzing end-to-end delays in most general multi-hop networks is a daunting task since they rely on routing protocols providing different routes toward destinations, different MAC layers and packet scheduling strategies, which affect delays experienced by messages traveling through a network. Estimating e2e delay becomes really challenging in case of wireless sensor networks (WSN) where topologies are ad-hoc and resources are limited.

Some recent work tried to better capture the WSN behavior by providing different analytic models. In [6], by considering a linear network (representing a path in the WSN), the e2e delay is analyzed using queueing theory for TDMA and slotted ALOHA MAC protocols. Each node is modeled by a GI/Geo/1 queue and the dependance between neighboring nodes is characterized by a correlation parameter. This result

\footnotetext{
${ }^{0}$ This work was partially supported by Quasimodo project under No. ANR 2010 INTB 020601 in France and No. NSFC 61061130563 in China
}

is however difficult to be used for analyzing general WSN. In [10], the authors proposed a more general framework for including both channel, MAC and routing characteristics in their analysis. By considering the CSMA/CA MAC protocol, each node is modeled by a Geom/PH/1/M queue where $\mathrm{PH}$ (Phase-Type) follows the distribution in [7]. The e2e delay distribution is obtained.

More recently, in [4], the authors presented a more general theoretic framework based on frequency domain analysis (i.e. generating function like Laplace transform) for estimating e2e delay distribution within a multi-hop network. They have defined several network structures. Taken as building blocks to model a wide variety of network topologies. Even though this calculus framework proposes a set of building blocks that can be used as a starting point to compute the delay in more complex scenarios, this task is still difficult, mainly because of the following factors. 1) it is in general very hard to obtain the delay distribution function of a network node; 2) the theoretic framework assumes the independence of the distributions of the neighboring nodes, which is not verified in general; 3) the queue stability condition has not been explicitly given in [4], but it is obviously necessary.

Alternative approach is discrete event simulation capable of including more complex networks and different topologies. It is able to check the validity of theoretical results and it is a complementary method for estimating the end-to-end delay, in particular for those scenarios which grow in complexity. However, such results are usually inaccurate, requiring several developments to integrate specific models and heavily depends on the used simulation tool [5], [3]. Hence, our idea is to combine such methods, despite that all of them are usually used to estimate e2e delays, by merging their advantages. Simulation approaches are helpful to deal with complex scenarios despite of a low general property of their results. Such specificity of their results could be generalized by analytical modeling which provides enough flexibility to suit general characteristics of multi-hop networks including WSN.

In this paper, we explore the analysis framework introduced in [4] to validate its results regarding e2e delay distribution and compare them with queueing analysis and simulations 
by means of a two concrete scenarios. We have applied a statistical analysis on data sets obtained from the different approaches to obtain the Cumulative Density Function (CDF) of the e2e delay to quantify the probabilistic QoS guarantee of the network. Our contribution in this paper consists in analysing the local delay distribution of nodes when considering a queueing network and a MAC protocol. The local delay distribution helps us in determining the entire e2e delay since the proposed framework does not consider the delay behaviour when dealing with nodes that introduce queueing delay. Firstly, we introduce a scenario in which we model a queueing network which we simulated using Java Modeling Tool (JMT) [2]. Then we compared results with the ones obtained by the framework. Queueing based analysis helped us to find the node delay distributions that we then integrated in the framework. We also present a scenario in which we consider the implications of having a MAC layer protocol in order to show whether the framework is still valid or not when considering a more realistic case. In order to analyse this scenario, discrete event simulation was used by means of the Prowler simulation tool [9]. In both cases we have collected the simulated data, and after analysing it, we have obtained the empirical e2e delay distribution CDF of the packets traversing the network which we then used to compare with theoretical results.

In this paper we considered scenarios having low and medium traffic load. In [11], authors combine real-time theory and queueing theory to provide stochastic models for unreliable networks for the case of heavy traffic rate. Other works, in which independence assumption between nodes is considered, ultra-low traffic (the peak rate of each source does not exceed $5 \%$ of the total link capacity) is analysed.

The structure of this paper is as follows. Section II introduces existing modeling approaches of e2e in multi-hop networks with a focus on the framework presented in [4]. Section III presents our evaluation methodology regarding proposed scenarios and obtained results using different approaches: analytic, queueing analysis and discrete event simulation. Finally, Section IV concludes the paper and presents our future work.

\section{End-To-End Delay Modeling}

We begin by describing the two modeling approaches for estimating end-to-end delay: analytical analysis as described in [4] and traditional queueing based analysis.

\section{A. Frequency domain based analysis}

End-to-end delay distribution is usually analyzed in the time domain using mainly Markov models. However, in [4], authors developed a theoretical framework to calculate the end-toend delay in a networked system using frequency-domain modeling and analysis where they shown that their approach is more scalable and allows analysis of compositional networked systems. They convert the analysis from time domain to frequency domain based on Laplace Transform. After getting the analysis results in frequency domain they convert the results back to time domain by means of the Inverse Laplace
Transform. This approach for computing the delay distribution converts the convolution calculation to multiplication, simplifying the calculation greatly. The idea consists in representing a network as a graph with a set of nodes and links between them and then to assign the reliability function, which could be tipically the packet delay probability distribution, to each of the components (nodes and links) in the network. They define four common building blocks and they analyze the distribution of each of them in order to take them as a start point for calculating the delay distribution in more complex scenarios: a serial structure, a parallel structure, circular structure and a back-up structure. Complex scenarios are then represented by one or a combination of these building blocks. A summary of the notation used by the calculus framework is presented in Table I. Once a scenario is specified they proceed to calculate the system e2e delay distribution by means of the Reliability Adjacency Matrix. This matrix represents a one hop delay relation of any pair of nodes. Thus, as shown in [4], for any nodes $v_{i}, v_{j}$ :

$$
A_{i, j}= \begin{cases}0, & \text { no direct connection; } \\ p_{i j} e_{i j}(s), & \text { direct connection; } \\ p_{i k} e_{i k}(s) \cdot p_{k j} e_{k j}(s), & \text { serial structure; } \\ \sum_{k} p_{i j k} e_{i j k}(s), & \text { k-parallel structure; } \\ \frac{p_{i j} e_{i j}(s)}{1-p_{i i} e_{i i}(s)}, & \text { circular structure; } \\ \min \left\{e_{i j 1}(s), e_{i j 2}(s) \cdots e_{i j k}(s)\right\}, & \text { k-backup structure; }\end{cases}
$$

\begin{tabular}{|c|l|}
\hline Notation & Meaning \\
\hline$e_{i j}$ & a direct link between $v_{i}$ and $v_{j}$ \\
\hline$p_{i j}$ & $\begin{array}{l}\text { the probability of selecting the link } e_{i j} \text { for going from } v_{i} \text { to } \\
v_{j}\end{array}$ \\
\hline$e_{i j k}$ & set of $k-$ parallel links from node $v_{i}$ to $v_{j}$ \\
\hline$p_{i j k}$ & $\begin{array}{l}\text { in a parallel structure means the probability of choosing the } \\
e_{i j k} \text { link }\end{array}$ \\
\hline
\end{tabular}

TABLE I: Table of notations of the reliability calculus framework.

Then, they define matrix operations according to the network topology and the nature of the protocols. In this way, they define the Reliability Adjacency Matrix in $r$ hops as $A^{r}$ which describes the end-to-end communication delay distribution along all links between any two components in the given system within $r$ hops. Examples on this can be found in [4]. The Reliability Adjacency Matrix allows to get the frequencydomain expression of the end to end communication function which is then converted to a general form expression. Finally, they apply the Inverse Laplace Transform to get the end to end communication delay distribution function in time domain. They also provide a hierarchical approach to get the e2e delay distribution in large-scale networks.

\section{B. Queueing network based analysis}

In [4], authors propose a way to compute the end to end delay in a set of predefined building blocks. However, none of the provided examples shows how to deal with queueing delay in nodes and how this delay influences in the end-to-end computation. A widely used method to analyse communication 
delays is queueing networks based modelling [1]. This method provides a probabilistic estimation of the end-to-end delays regarding an arrival model of messages and a service time model to process these messages by nodes. We introduce two scenarios in which we take into account, firstly, the queueing delay in nodes within a Jackson Network [1] where Jackson's results were applied, and then the queueing delay when considering a MAC layer protocol.

Jackson's Theorem states that in a queueing network having $K$ nodes and satisfying that

- Each node consists of $c_{i}$ identical exponential servers with service rate $\mu_{i}$

- External messages arrive from outside at queue $i$ in a Poisson pattern with rate $\gamma_{i}$

- Once message is served in queue $i$ it goes to queue $j \in$ $1,2, \ldots, K$ with probability $p_{i}$ or leave the network with probability $1-\sum_{j=1}^{K} p_{i j}$

then the average arrival rates $\lambda=\left(\lambda_{1}, \lambda_{2}, \ldots, \lambda_{K}\right)$ to each queue can be computed as

$$
\lambda=\gamma(I-P)^{-1}
$$

where matrix $I$ is the identity matrix, $P$ is the transition matrix from one queue to another in terms of probability, i.e. $P_{i j}$ represents the probability of transition from queue $i$ to queue $j$ and vector $\gamma$ represents the external message arrival rate for each queue $i$. Furthermore, each queue $i$ behaves as if it is an independent $M / M / c_{i}$ queueing system with average arrival rate $\lambda_{i}$. With these ideas in mind we have analysed the queueing delay in a Jackson network and we have computed the entire end-to-end delay by merging the framework calculus together with these queueing delay results.

\section{Comparative Evaluation}

\section{A. Methodology}

In order to verify other applicability of the theoretical approach presented in [4] (called hereafter framework) we have defined two empirical experiments using two simulator tools: JMT, which is a performance evaluation suite based on queueing network models, and Prowler which runs under MATLAB and provides an easy way of application prototyping with nice visualization capabilities. We have defined a Jackson's scenario which we have tested using JMT and we have compared the results with the ones obtained by the framework. The scenario is a linear network with nodes in tandem having certain arrival rate $\gamma$ in which nodes or queues introduce a delay component due to the queueing delay before transmitting the messages toward the next node. A description and results for this scenario are shown in next section. We have also defined a network scenario in which our purpose is to compare the approach described in the framework [4] with an empirical example, that is to say, to show how close to a more realistic scenario the framework is. In this way, we have simulated the proposed scenario in Prowler simulator for two different cases of the workload: $\rho(0.1,0.5)$. Our idea is to use Prowler in order to take advantage of its layered architecture to introduce a MAC layer which is based on CSMA/CA. We have analysed the behaviour of this protocol and we then estimated the delay distribution function of links and nodes, which are the building blocks to use within the framework. The network topology is the same as the one we used for the JMT scenario but with different parameters. In order to compare the results we have plotted the results for both Prowler and framework considering different values of $\rho$. Next subsections describe in detail the evaluation results and our analysis.

\section{B. Results}

We show the results obtained by running the described scenarios in JMT and Prowler and we compare them with the theoretical results obtained in [4]. Both theoretical and empirical delay distributions are shown. For both scenarios, based on the kernel density estimation [8], we have estimated the probability density function of the empirical collected data and plotted the corresponding CDF together with the theoretical CDF.

a) JMT Scenario: In order to show how the framework behaves when dealing with queueing delay we have included a Jackson Network [1] scenario which we have ran using JMT simulation tool. Based on the Jackson's analysis shown in previous section and considering that queueing delay is not negligible and must be taken into account when analysing the local and entire delay, we introduce as a contribution, a queueing based analysis, which helped us in determining the local delay distribution, and the implications of adding it to the framework. We have applied Jackson's results explained before. Figure 1 shows the scenario we have analysed.

Nodes delay have exponential distribution with parameter

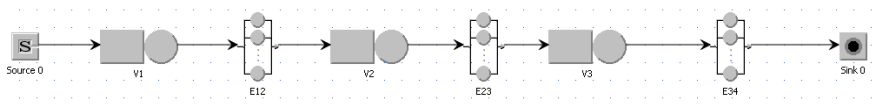

Fig. 1: Open queueing Jackson network based scenario.

$\mu=34$ while links delay have exponential distribution with parameter $\mu=1$. Messages arrive as Poisson process with parameter $\gamma=17$. We determined the local delay of each node as follows:

Since there are no feedback transitions, the matrix $P$ is

$$
\left(\begin{array}{lll}
0 & 1 & 0 \\
0 & 0 & 1 \\
0 & 0 & 0
\end{array}\right)
$$

Then, the $\gamma$ vector is ( $\left.\begin{array}{lll}17 & 0 & 0\end{array}\right)$. By applying equation (1) we obtained a $\lambda$-vector ( $\left.\begin{array}{lll}17 & 17 & 17\end{array}\right)$ which specifies the arrival rate $\lambda_{i}$ of messages for all the $i$ queues in the system. We then compute the sojourn time distribution for each queue which is exponentially distributed with parameter $\mu(1-\rho)$ where $\rho=\frac{\lambda}{\mu}$.

Once we have computed the nodes delay distribution $v_{i}(s)$ and the links delay distribution $e_{i j}(s)$, we computed the e2e delay 
by applying the serial formule from [4] as follows:

$$
\begin{aligned}
e_{s r c, s k}(s) & =\prod v_{i}(s) \cdot e_{i j}(s) \\
& =v_{1}(s) \cdot e_{12}(s) \cdot v_{2}(s) \cdot e_{23}(s) \cdot v_{3}(s) \cdot e_{34}(s)
\end{aligned}
$$

Results are shown in Figure 2.

b) CSMA/CA scenario: In order to provide a more realistic configuration we have defined a scenario based on an in tandem structure where we incorporate the effect of a MAC layer on each node. We have voluntarily chosen a simple scenario in order to compare both approaches. However, in [4], a set of network building blocks were defined and can be used to model more complex network topologies. In this scenario our idea is to incorporate attributes from real networks and specially regarding the MAC layer in which delay is affected by many parameters such as random backoff time, busy channels, collision errors, among others. Scenarios considered in [4] do not consider any MAC layer so we introduce this one in order to see how the e2e delay behaves in a simulator which emulates a real network system. We have used the CSMA/CA protocol implementation provided by the Prowler simulator. Messages arrive at node 1 as Poisson Process with parameter $\gamma$ and travels through node 2 and 3, which are relay nodes, until reaching the fourth and final node. Neighbor nodes are separated by a distance of 0.5 meters. The radio propagation model is shown below

$$
P_{\text {rec }, \text { ideal }}(d)=P_{\text {transmit }} \frac{1}{1+d^{\tau}}
$$

The fading effect is modeled by random disturbances in the simulator. The received signal strength from node $j$ to node $i$ is calculated from the propagation function by modulating it with random functions

$$
P_{\text {rec }}(i, j)=P_{\text {rec }, \text { ideal }}\left(d_{i j}\right) \cdot\left(1+\alpha\left(d_{i j}\right)\right) \cdot(1+\beta(t))
$$

A parameter $p_{\text {error }}$ determines the probability that a collision occurs when a message is sent from one node to another. Collisions also happen in the receiver side if a message is sent to it and it has already started to receive another message. In case a collision occurs the message is dropped. We have set $p_{\text {error }}=0$ so collision may only occurs in the reception process. The channel bitrate is $40 \mathrm{Kbps}$. Finally, the maximum queue length for each node is 20 . As we will see further, this queue length is enough to guarantee that no messages are dropped due to full queues. The maximum number of attempts in backoff mode is 10. Messages in backoff that reach this threshold are dropped. Parameters are detailed below. We proceed now to compute the estimated service time of each node considering the parameters that we have previously defined. In particular, the estimated service time depends on the packet size, channel bit rate and also the waiting and backoff times. The real delay for sending a packet is:

Delay $_{p k}=p($ waiting time + packetTime $)+(1-p)(\text { backoff time })^{k}$

where $k$ is the number of backoff which has geometric distribution, $p$ is the probability that a node finds the medium

\begin{tabular}{|c|l|}
\hline Notation & Meaning \\
\hline$P_{\text {rec }, \text { ideal }}(d)$ & ideal reception signal strength. \\
\hline$P_{\text {transmit }}$ & transmission signal power. \\
\hline$d$ & $\begin{array}{l}\text { distance between transmitter } i \text { and the receiver } \\
j .\end{array}$ \\
\hline$\tau$ & decay parameter, with value 2 in our case. \\
\hline$P_{r e c}(i, j)$ & real reception signal strength. \\
\hline$\alpha$ & random variable $N\left(0, \sigma_{\alpha}\right)$. Distance dependent. \\
\hline$\beta$ & random variable $N\left(0, \sigma_{\beta}\right)$. Time dependent. \\
\hline$\sigma_{\alpha}$ & 0.45 \\
\hline$\sigma_{\beta}$ & 0.02 \\
\hline waiting time & $5 \mathrm{~ms}+3.2 \mathrm{~ms} * \mathrm{U}(0,1)$ \\
\hline backoff time & $2.5 \mathrm{~ms}+0.7 \mathrm{~ms} * \mathrm{U}(0,1)$ \\
\hline max queue length & 20 \\
\hline max backoff attempts & 10 \\
\hline
\end{tabular}

TABLE II: Table of notations of the propagation and fading models.

idle when it attemps to transmit and packetTime is the time taken for the channel to send all packet bits. Since the packet length is 960 bits and having a channel bitrate of $40 \mathrm{Kbps}$, the time taken for a node to transmit a packet is packetTime $=24 \mathrm{~ms}$ (960/40Kbps). Since we don't have an estimation of parameter $p$ we have taken a more simplistic assumption when modeling the node delay. Considering that the delay in 5 has some constant components we have taken this constant to model the links delay. We have taken the packetTime and the constant part of both waiting time and backoff time as the parameter to model the links having constant distribution with parameter $m=0.029 m s(5 m s+2,5 m s+24 m s)$. Nodes where modeled with exponential service distribution as well but taking the random part of waiting time and backoff time. In this case, the parameter $\mu=256\left(=\frac{1}{3.2 m s+0.7 m s}\right)$. Since it is also an in tandem scenario we consider the serial structure formule 2 for calculating the e2e delay distribution. We have tested this scenario taking two different parameters of $\rho$, namely $\rho=0.1$ and $\rho=0.5$ as shown in Figures 3 and 4. Considering that messages arrive as Poisson process and since nodes has exponential service time, we have modelled them as a $\mathrm{M} / \mathrm{M} / 1$ queue. In this case, the Laplace transform of the delay in nodes is:

$$
\operatorname{Delay}_{v}=\frac{\mu-\gamma}{(s+\mu-\gamma)}
$$

On the other hand, we have considered constant delay in links. Taking the assumption that the output rate of $\mathrm{M} / \mathrm{M} / 1$ is also Poisson we have modelled the links as a M/D/1 queue where the service time has constant parameter $\mu=\frac{1}{5 m s+2,5 m s+24 m s}$. In this case, the Laplace transform for the links delay is

$$
\operatorname{Delay}_{e}=\frac{s(1-\rho) e^{-s m}}{s-\gamma+\gamma e^{-s m}}
$$

where $\rho=\frac{\gamma}{\mu}$. We have mentioned that during the simulation there are messages that were dropped. These messages are those that reach the maximum number of attempts (backoff threshold parameter) to find the channel idle. That means that the real arrival rate to each queue depends on the number of dropped messages. The effective arrival rates for the case $\rho=0.5$, together with some other results, are summarized in 
Table III. In the case of $\rho=0.1$ there are no drops so the parameter $\gamma$ in this case is the same for all queues. The first

\begin{tabular}{|c|c|c|c|c|}
\hline Queue & \% Drops & \% Drops Full Queue & $\gamma_{i}$ & Avg. Backoff \\
\hline 1 & 12 & 0 & 17 & 5.1 \\
\hline 2 & 22 & 0 & 14.9 & 5.6 \\
\hline 3 & 12 & 0 & 11.7 & 4.9 \\
\hline
\end{tabular}

TABLE III: Simulation Summary.

column in Table III represents the queue number, the second column shows percentage of messages dropped, the third one the percentage of messages dropped due to full queues, $\gamma_{i}$ is the effective arrival rate to each node and the last column shows the average number of attempts to find the channel idle that messages have experienced for each queue during the simulation. As we can see, the third column confirms that the queue length parameter shown in Table II is enough to consider no message loss due to full queues. By applying 6 and 7 with the corresponding $\gamma$ and $\mu$ parameters we proceeded to compute the delay of each node and link in the system. Then, e2e delay holds by applying 2 . A discussion of the results is presented in next section.

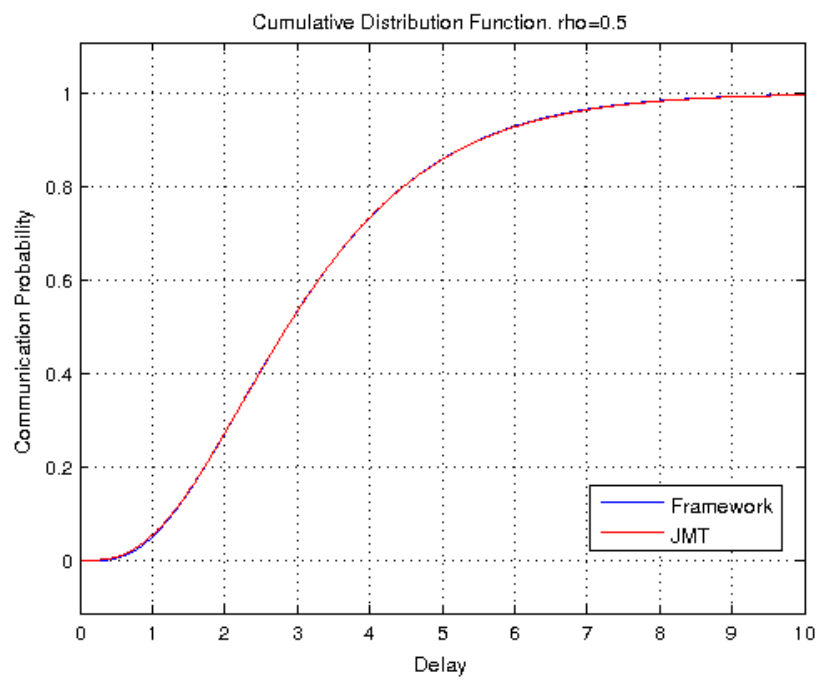

Fig. 2: Comparison between Framework and JMT with $\rho=$ 0.5 .

\section{Discussion}

Previously, we have presented two scenarios and we have shown the results by comparing the CDF of both theoretical and empirical curves. For the Jackson scenario implemented in JMT, Figure 2 shows that both curves fit perfectly so empirical and theoretical results are the same for this queueing network scenario. For the Prowler scenario, we have taken some considerations in order to simplify the model. In particular, we have considered a slightly different approach for determining the delay introduced by the CSMA/CA since we do not have probability $p$ that a node finds the medium idle when it attemps to transmit. An approach for solving this can be found in

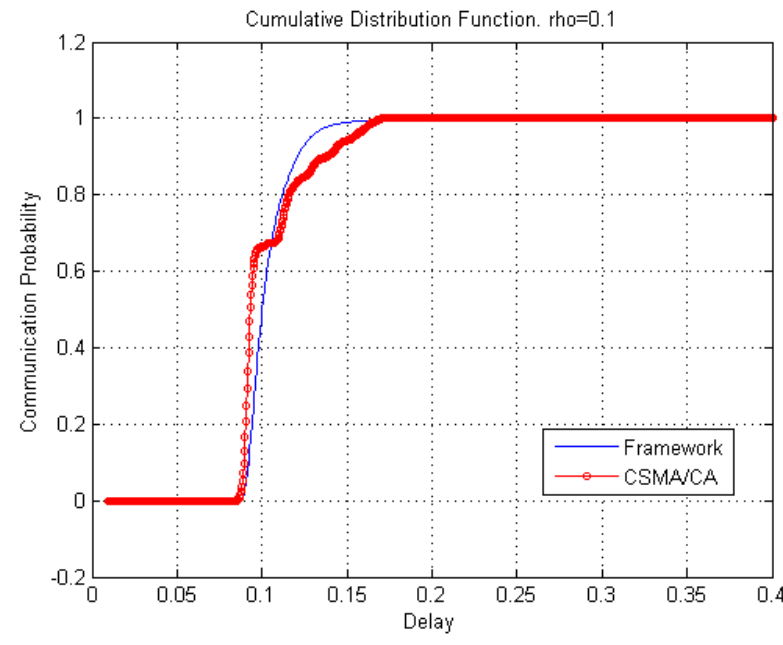

Fig. 3: Comparison between Framework and CSMA/CA with $\rho=0.1$.

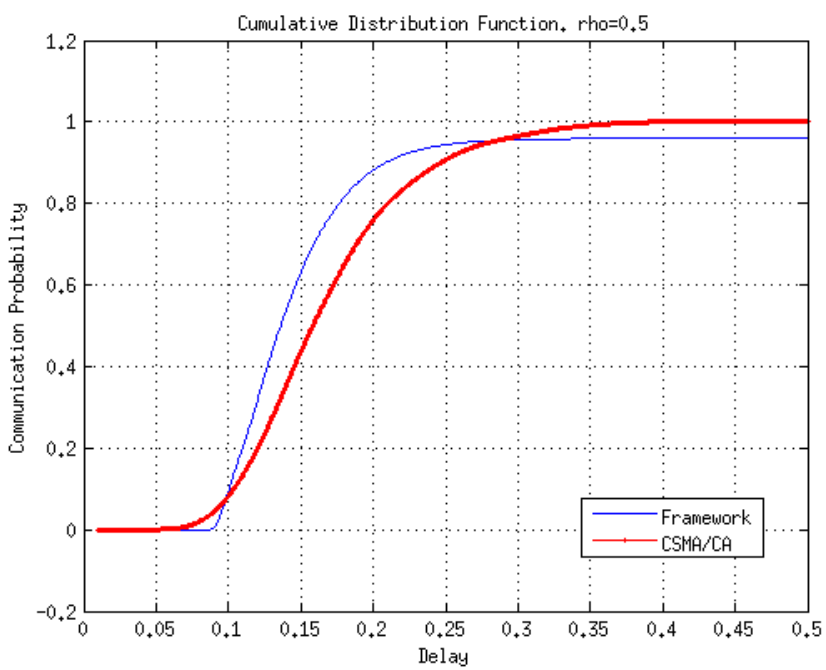

Fig. 4: Comparison between Framework and CSMA/CA with $\rho=0.5$.

[12]. In this way, we have done a simplification in our model when analysing the delay introduced by CSMA/CA. As we mentioned before, the probabilistic QoS only requires that the e2e delay should not exceed a given threshold with certain probability. So this approximation still gives significant result. We can see from Figure 3 and 4 that, for these two cases with low and medium traffic load, for a given threshold, say $250 \mathrm{~ms}$, the theoretical curves provide satisfying e2e delay meet probability $P[e 2 e$ delay $>250 \mathrm{~ms}]$. Table IV summarize these results.

In the case of $\rho=0.1$ where the load traffic is low and the channel is almost all the time idle, the extra delay due to the backoff process can be considered almost negligible and thus, the model we have done for estimating the delay in node is not so far from the empirical one as shown in Figure 3. For 


\begin{tabular}{|l|l|}
\hline Scenario $(\rho=0.1)$ & $P[$ e2e delay $>250 \mathrm{~ms}]$ \\
\hline Framework & 0 \\
\hline CSMA/CA & 0 \\
\hline Scenario $(\rho=0.5)$ & $P[e 2 e$ delay $>250 m s]$ \\
\hline CSMA/CA & 0.093 \\
\hline Framework & 0.056 \\
\hline
\end{tabular}

TABLE IV: e2e delay meet probability.

the case of $\rho=0.5$, an increase in the number of times a message enters in backoff is expected since the probability of finding the channel idle is lower than the case of $\rho=0.1$. This can be seen in Figure 4 where both curves are not so close to each other. However, results are still acceptables taking into account the e2e delay meet probability.

\section{CONCLuSions}

In this paper we have presented an empirical support and evaluation of a theoretical framework [4] for estimating endto-end delay distribution in a multi-hop network. The explored framework relies on frequency domain analysis and provides a set of compositional building blocks to model complex networks. We have developed two scenarios, one in which we analysed the framework behaviour when considering a queueing network and the second one in which we analysed the delay in nodes when considering the CSMA/CA protocol. Our study shows that the proposed analytical approach is able to estimate e2e delay distribution when considering queueing delay in nodes for the case of low and moderate traffic. Comparing to the simulation approach, which may be too complex for very large scale networks, the analytical framework may be used for getting a good approximation when considering low and medium traffic load.

As future work, we plan to extend the approach by analysing the e2e delay in more realistic scenarios, i.e. by including a more refined analysis in the delay introduced by MAC protocols, as the one explained in [10], and also the delay introduced by routing protocols as well.

\section{REFERENCES}

[1] Arnold O. Allen. Probability, statistics and queueing theory - with computer science applications (2. ed.), chapter 5. Academic Press, 1990.

[2] Marco Bertoli, Giuliano Casale, and Giuseppe Serazzi. Jmt: performance engineering tools for system modeling. SIGMETRICS Perform. Eval. Rev., 36(4):10-15, 2009.

[3] E. Egea-Lopez, J. Vales-Alonso, A. Martinez-Sala, P. Pavon-Mario, and J. Garcia-Haro. Simulation scalability issues in wireless sensor networks. Communications Magazine, IEEE, 44(7):64 - 73, july 2006.

[4] Wenbo He, Xue Liu, Long Zheng, and Hao Yang. Reliability calculus: A theoretical framework to analyze communication reliability. In Proceedings of the 2010 IEEE 30th International Conference on Distributed Computing Systems, ICDCS '10, pages 159-168, Washington, DC, USA, 2010. IEEE Computer Society.

[5] D. Kotz, C. Newport, R.S. Gray, J. Liu, Y. Yuan, and C. Elliott. Experimental evaluation of wireless simulation assumptions. In Proceedings of the 7th ACM international symposium on Modeling, analysis and simulation of wireless and mobile systems, pages 78-82. ACM, 2004.

[6] M. Haenggi M. Xie. Towards an end-to-end delay analysis of wireless multi-hop networks. Ad Hoc Networks, 7:849-861, 2009.

[7] M.F. Neuts. Matrix-Geometric solutions in stochastic models: an algorithmic approach. Dover Publications Inc., 1981.
[8] Emanuel Parzen. On estimation of a probability density function and mode. The Annals of Mathematical Statistics, 33(3):pp. 1065-1076, 1962.

[9] G. Simon, P. Volgyesi, M. Maroti, and A. Ledeczi. Simulation-based optimization of communication protocols for large-scale wireless sensor networks. In Proceedings of IEEE Aerospace Conference, volume 3, pages 1339-1346, Big Sky, MT, USA, 2003.

[10] S. Goddard Y. Wang, M.C. Vuran. Cross-layer analysis of the end-toend delay distribution in wireless sensor networks. In IEEE Computer society, editor, 30th IEEE Real-Time Systems Symposium, pages 138147, December 2009.

[11] Shu-Ngai Yeung and John Lehoczky. End-to-end delay analysis for real-time networks. Real-Time Systems Symposium, IEEE International, $0: 299,2001$

[12] Jiazhen Zhou and Kenneth Mitchell. A scalable delay based analytical framework for csma/ca wireless mesh networks. Comput. Netw., 54:304318, February 2010. 